\title{
Role of dipole-dipole interactions for hyperthermia heating of magnetic nanoparticle ensembles
}

\author{
C. Haase ${ }^{*}$ and U. Nowak \\ Department of Physics, University of Konstanz, D-78464 Konstanz, Germany
}

\begin{abstract}
For clinical hyperthermia treatment the heating efficiency of magnetic nanoparticle ensembles is a crucial element. Using efficient algorithms, this heating is studied numerically with a focus on the effects of dipole-dipole interparticle interactions. For the time evolution of realistically modeled systems an approach based on the Landau-Lifschitz-Gilbert equation of motion with Langevin dynamics is taken. Our results suggest a widely negative influence of dipole-dipole interactions on the heating power of nanoparticles. However, considering ensembles within a fixed, given sample volume an optimal particle density exists. The presented results may have important implications for the medical use of magnetic hyperthermia treatment.
\end{abstract}

PACS number(s): 87.85.Rs, 75.50.Tt, 75.60.Ej, 87.19.Pp

\section{INTRODUCTION}

Magnetic-fluid hyperthermia, a new experimental cancer treatment, uses the heating of magnetic nanoparticles in an alternating magnetic field as a therapeutic measure to selectively heat and destroy tumor cells within the human body. For such a treatment magnetic nanoparticles in a solvent liquid are injected into the bloodstream and can be navigated by external magnetic field gradients and/or biological receptor coatings on the particle surface to the target region. The particles accumulate therefore in the tumor cells and can then heat them selectively until destruction sets in.

Various studies have already been performed on this subject, both experimentally and theoretically. Experiments range from the physical studies of the nanoparticles and the liquid ${ }^{1,2}$ to medical studies on toxicity, ${ }^{3}$ targeting ability, ${ }^{4,5}$ intracellular mobility, ${ }^{6}$ and the effectiveness of the heating. ${ }^{7,8}$ Theoretically, most works deal with the general description of the particle assemblies, both analytically ${ }^{9,10}$ and numerically. ${ }^{11,12}$ Here the general focus is on the optimization of the heating power and its dependence on particle properties, such as their size or the material parameters. ${ }^{13}$

Two fundamental values that influence the heating power of particles directly are the frequency $f$ of the alternating magnetic field and its amplitude $B_{0}$. Due to the nonselective heating effects of eddy currents in the human tissue these values must, however, be limited in size to assure a safe treatment for the patient. ${ }^{14}$ Aside from the small contribution of eddy currents the heating of magnetic particles may be caused by magnetic hysteresis losses or by viscous friction losses that occur with a Brownian particle rotation. The latter effect is, however, neglectable in a realistic treatment scenario since particles are strongly immobilized in the human tissue. ${ }^{6}$

The heating that is linked to the system hysteresis is caused by magnetic relaxation and does not need to be distinguished from heating trough Néel relaxation losses. ${ }^{15}$ Both loss processes are, for one field cycle, fully defined by the area $A_{\text {Hyst }}$ that is enclosed in the hysteresis loop of the particle ensemble. ${ }^{16}$ The most commonly used quantity that reflects the heating power of a particle ensemble is the specific absorption rate (SAR, W/kg), which is defined as the heating power per particle mass. For a hysteresis loss process, this value is given as SAR $=\frac{A_{\mathrm{Hys}} f}{\rho}$, where $\rho$ denotes the density of the magnetic material. When $f$ and $B_{0}$ are kept constant at their medically safe values, it is sufficient to discuss a normalized hysteresis area $A=\frac{A_{\text {Hyst }}}{4 B_{0} M_{\mathrm{s}}}$ that defines the SAR for a certain material with saturation magnetization $M_{\mathrm{s}}$ and density $\rho$.

An important question that has recently found increasing attention is the influence of the unavoidable dipole-dipole interactions among the particles. Present discussions are still not fully conclusive on whether they cause an increase or a decrease of the SAR. To the best of our knowledge the latest results on this subject point, however, to a reduced SAR. ${ }^{17}$ As the strength of dipole-dipole interactions depends strongly on the particle density, this interaction may very well define an optimal density that has to be chosen for a maximized heating power. We suggest considering a volume specific absorption rate (VSAR, $\mathrm{W} / \mathrm{m}^{3}$ ) that denotes the heating power in a fixed sample volume encapsulating a varying amount of nanoparticles. Optimizing this quantity would be of great importance for practical studies that focus on increasing particle densities in the tumor volume through modern targeting methods.

In order to contribute to the discussion of dipole-dipole effects in hyperthermia treatment we will employ an advanced computer model that is able to simulate nanoparticle systems with many of their practical features, such as a particle-size distribution, random particle position, random orientation of the easy axes, and dipole-dipole interaction. We will show that optimal particle densities exist for a maximization of the VSAR.

\section{MODELING AND SIMULATION APPROACH}

Within the computational approach used the magnetization dynamics of each particle is represented by the Landau-Lifshitz-Gilbert (LLG) equation of motion, ${ }^{18}$ including Langevin dynamics for the consideration of thermal properties,

$$
\dot{\mathbf{S}}_{i}=-\frac{\gamma}{\left(1+\alpha^{2}\right) M_{\mathrm{s}} V_{i}} \mathbf{S}_{i} \times\left[\mathbf{H}_{i}(t)+\alpha \mathbf{S}_{i} \times \mathbf{H}_{i}(t)\right] .
$$

In this equation $S_{i}$ are unit vectors pointing into the direction of the magnetization. The coefficient $\gamma$ denotes the absolute value of the gyromagnetic ratio, and $\alpha$ is the dimensionless Gilbert damping constant. The effective field $\mathbf{H}_{i}(t)=\zeta_{i}(t)-\partial \mathcal{H} / \partial \mathbf{S}_{i}$ is defined through the Hamiltonian

$$
\mathcal{H}=\mathcal{H}_{\text {anis }}+\mathcal{H}_{\text {field }}+\mathcal{H}_{\text {dipol }}
$$


and a thermal noise contribution $\zeta_{i}(t)$ with

$$
\begin{aligned}
\left\langle\zeta_{i}(t)\right\rangle & =0, \\
\left\langle\zeta_{i v}(t) \zeta_{j \varphi}\left(t^{\prime}\right)\right\rangle & =\delta_{i, j} \delta_{v, \varphi} \delta\left(t-t^{\prime}\right) k_{\mathrm{B}} T \mu_{\mathrm{s}} \frac{2 \alpha}{\gamma} .
\end{aligned}
$$

In Eq. (3) the Latin indices refer to different particles, whereas the Greek ones denote Cartesian coordinates.

In this approach the common approximation of singledomain magnetic particles is used with a magnetic moment $\mu_{i}=M_{\mathrm{S}} V_{i} \cdot \mathbf{S}_{i}$ that is proportional to the particle volume $V_{i}$ and a constant saturation magnetization $M_{\mathrm{s}}$. The Hamiltonian defining the effective field in Eq. (1) includes contributions from a uniaxial anisotropy with anisotropy constant $K$,

$$
\mathcal{H}_{\text {anis }}=-K \sum_{i} V_{i}\left(\mathbf{a}_{i} \cdot \mathbf{S}_{i}\right)^{2},
$$

an alternating, external magnetic field,

$$
\mathcal{H}_{\text {field }}=-\mathbf{B}(t) \cdot M_{\mathrm{s}} \sum_{i} V_{i} \mathbf{S}_{i},
$$

and dipole-dipole interactions,

$$
\mathcal{H}_{\text {dipol }}=-\frac{\mu_{0} M_{\mathrm{s}}^{2}}{4 \pi} \sum_{i<j} V_{i} V_{j} \frac{3\left(\mathbf{S}_{i} \cdot \mathbf{e}_{i j}\right)\left(\mathbf{e}_{i j} \cdot \mathbf{S}_{i}\right)-\mathbf{S}_{i} \cdot \mathbf{S}_{j}}{r_{i j}^{3}} .
$$

In addition to an individual, random orientation of its easy axis each particle is also equipped with an individual volume. Over the entire system the particle radii $r$ are distributed according to a standard lognormal probability density function: ${ }^{19}$

$$
F_{\mathrm{P}}(r)=\frac{1}{r \sigma \sqrt{2 \pi}} \exp \left(-\frac{(\ln r-\theta)^{2}}{2 \sigma^{2}}\right) .
$$

The coefficients $\theta$ and $\sigma$ define the average radius $r_{\text {mid }}$ and the relative standard deviation $\phi$ through $r_{\text {mid }}=\exp \left(\theta+\frac{1}{2} \sigma^{2}\right)$ and $\phi=\sqrt{\exp \left(\sigma^{2}\right)-1}$. Even though $r_{\text {mid }}$ is kept constant, the average particle volume $V_{\text {mid }}$ is changed for different distribution widths $\phi$.

Due to the numerical complexity of the dipole-dipole interactions an algorithm based on fast-Fourier-transformation methods is used for its evaluation. ${ }^{20}$ As this algorithm requires a regular lattice for its application, the modeled disorder is introduced by distributing relatively few particles on a very fine regular mesh. Each particle is set to fully occupy the space defined by its individual volume $V_{i}$ and is placed with a fixed position and orientation of its easy axis. Through this approach disordered particle systems with up to $10^{5}$ particles can be simulated over time scales of the order of $10^{-5} \mathrm{~s}$. Part of the remanent state of a sample system is shown in Fig. 1.

As an integration scheme for Eq. (1) a standard Heun method is used. ${ }^{20}$ In order to determine the validity of our approach, first, results neglecting the dipole-dipole interactions were compared with various standard problems that possess analytical solutions. The more complex simulations including dipole-dipole interactions are performed for systems at $T=300 \mathrm{~K}$ with constant average particle sizes of $r_{\text {mid }}=$ $15 \mathrm{~nm}$. The saturation magnetization is set to $M_{\mathrm{s}}=800 \mathrm{kA} / \mathrm{m}$, and the anisotropy constant is set to $K=10 \mathrm{~kJ} / \mathrm{m}^{3}$. Such particles are in the transition between superparamagnetic and
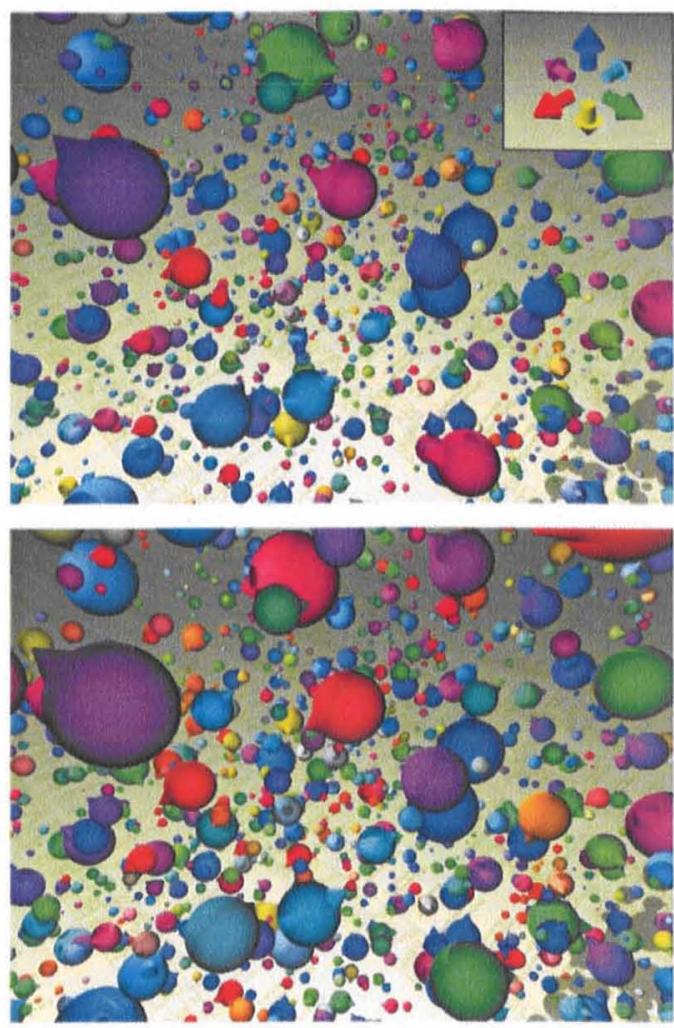

FIG. 1. (Color online) Illustration of the remanent states of two simulated particle ensembles. The lower system is a more dense version of the upper one with slightly lower remanence. The color coding as given in the inset in the upper plot visualizes the current magnetization orientation, which is also indicated by the peak on each particle. The depicted particle size corresponds to the assigned individual particle volume $V_{i}$. In this case the lognormal size-distribution width is defined by $\phi=0.6$ [see Eq. (7)].

Stoner-Wohlfarth-like behavior. They are expected to be the best candidates for an efficient magnetic heating under realistic conditions. ${ }^{15}$

The influence of the Gilbert damping parameter $\alpha$ is investigated for low- and high-damping cases. Since no qualitative change in the results is found (see Fig. 4), the majority of the simulations are performed in a high-damping limit with $\alpha=1$. For the same reasons also the frequency is chosen to be up to two orders of magnitude higher then under experimental conditions where $f$ is usually set in the region of $100 \mathrm{kHz}$. Neither of these simplifications is strictly necessary, but they serve to significantly reduce computation times. The magnetic field amplitude is set to $B_{0}=20 \mathrm{mT}$, a practically used value. ${ }^{14}$

The typical system size in the range from 2000 to 8000 particles is small compared to realistic systems but has proven to show reliable behavior. Within the limits of our computational means larger systems did not show a qualitative change in the results. 


\section{RESULTS}

Using the computer model that was introduced in the previous section, the influence of dipole-dipole interactions on the heating power is investigated for many different cases. The heating power is characterized by the reduced hysteresis area $A$ as it was introduced in Sec. I. In addition to the variation of particle concentration $c=V_{\text {particle }} / V_{\text {system }}(\%)$ (where $V_{\text {system }}$ is the volume of the entire sample encapsulating all magnetic nanoparticles and $V_{\text {particle }}=\sum_{i} V_{i}$ is the total volume of the particles), the influence of different sample shapes and different spatial distributions is studied as well. The heating efficiency of the particles is discussed per particle mass as well as per sample volume.

The most basic effect of dipole-dipole interactions on the hysteresis loops can be seen in Fig. 2. Three simulated curves are shown for different particle concentrations $c$. Note that the limit $c \rightarrow 0$ corresponds to vanishing dipole-dipole interaction since we keep the volume of the particles constant, and the limit $c \rightarrow 0$ hence refers to an increasing system volume and, with that, to an increasing distance between particles. Both the remanence and the coercive field are reduced for an increasing particle concentration. It is interesting to note that for higher concentrations no saturation is reached and only an inner hysteresis loop can be seen. The hysteresis area $A$ and therefore the system's SAR are clearly reduced:

This reduction is shown in more detail in Fig. 3. Here different particle-size distributions and sample shapes are analyzed. Note that the term sample shape here refers to the overall shape of the the entire ensemble of particles, not to the shape of the individual particles. For $\phi=0$ all particles have identical size, while $\phi=0.8$ corresponds to a lognormal distribution of finite width. The sample shape is generally defined by the ratio $\mathcal{R}_{\mathrm{x}, \mathrm{y}}=L_{z}: L_{x, y}$ of its length in the field direction versus its orthogonal width. The decrease in hysteresis area $A$ for an increasing particle concentration $c$ can be seen for all systems. For only the elongated sample a limited benefit is produced by the dipole-dipole interactions at small concentrations. This effect is due to the shape anisotropy of the elongated sample leading to an increased coercivity and hence hysteresis area. The influence of the particle-size distribution is less pronounced.

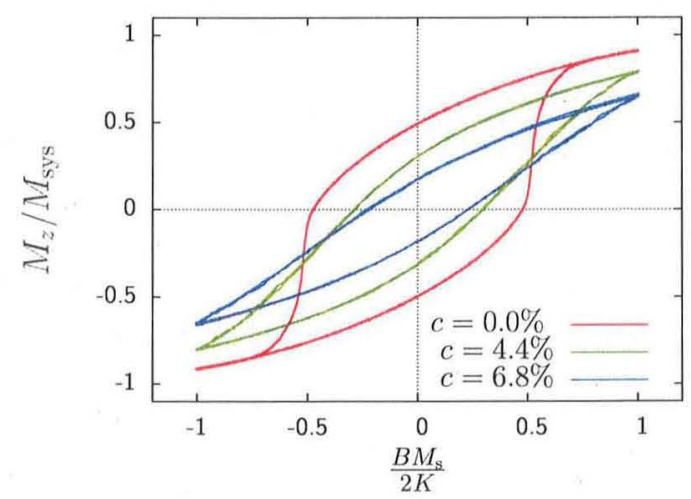

FIG. 2. (Color online) Simulated hysteresis curves for different particle concentrations $c$. The remanence and the coercivity are clearly reduced for higher concentrations.

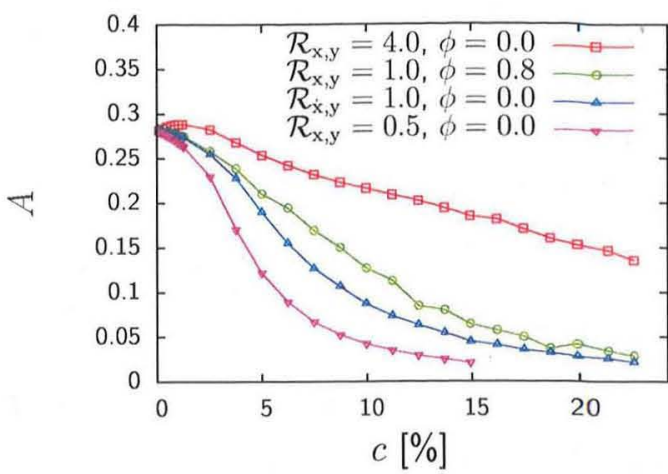

FIG. 3. (Color online) Reduced hysteresis area $A$ vs particle concentrations $c$. This dependence is shown for different sample shapes and size distributions

In comparison to the results of Fig. 3 it can be seen in Fig. 4 that the former frequency and damping values $(f=10 \mathrm{MHz}$ and $\alpha=1$ ) produce only small alterations to simulation results obtained for more realistic values of $f$ and $\alpha$. The qualitative behavior of the system for increasing particle density remains the same.

When increasing the particle concentration in a system, it is further important to consider the spatial distribution of particles. When used for hyperthermia treatment, magnetic nanoparticles will have a functionalized nonmagnetic coating that protects the particles, prevents particle agglomeration, and may be equipped with biological targeting receptors. If no or very little coating exists, particles will be able to randomly form dense clusters even when diluted significantly in a solvent, while a thicker coating might well prevent this cluster formation. The effect such a difference in the spatial distribution has on the influence of dipole-dipole interactions is shown in Fig. 5. The simulated hysteresis curves are, in one case, for a system where particles are generally placed with a larger distance to other particles (repellent particles) and, in another case, for a system where particles are placed randomly and thus may statistically form denser clusters (free particles). These distinct spacial distributions are created using different system-modeling algorithms. Free particles are simulated by

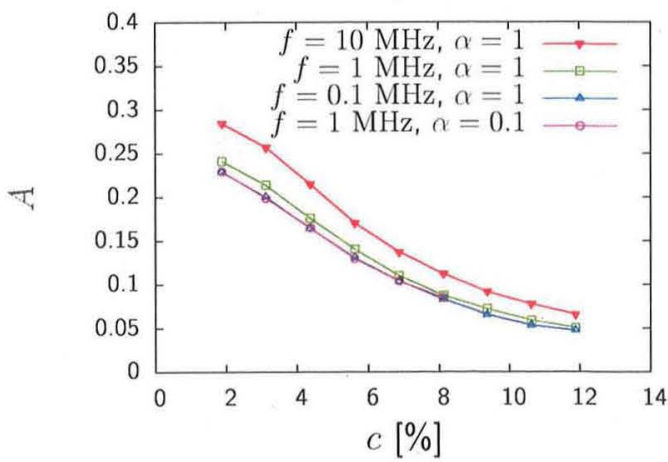

FIG. 4. (Color online) Reduced hysteresis area $A$ vs particle concentration $c$. The effects of different frequencies $f$ and damping constants $\alpha$ are shown. Qualitatively, the same dependence on particle density can be seen for all cases. 


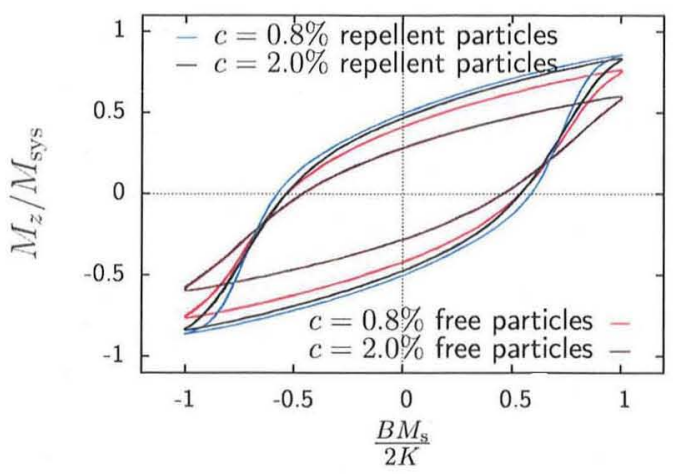

FIG. 5. (Color online) Simulated hysteresis curves for systems with different spatial distribution of the particles. While the repellent particles have larger interparticle distances, the free particles are randomly arranged. For both cases two different particle concentrations $c$ are shown.

distributing a fixed amount of particles on a fine mesh of variable size. The elementary mesh constant is chosen to be small enough that two particles may be "touching" even for small concentrations. An overlap of two particles is prohibited by a positioning algorithm that prevents particles from being placed too close to each other. Repellent particle systems are created by expanding the mesh of a very dense free-particle system. An appropriate renormalization of the elementary mesh constant is used to lower the particle concentration to any appointed value. It is evident from the results that for the same particle concentration $c$ significantly stronger dipole-dipole effects occur for free particles than for repellent particles. That means that clusters of particles with relatively small distances govern the effects of the dipole-dipole coupling.

In contrast to the focus that has so far been on the hysteresis area $A$ and therefore on the heating power per particle mass (SAR), in Fig. 6 we investigate the heating power per sample volume (e.g., size of the tumor). All former results showed decreasing hysteresis areas for (strongly) increased particle concentrations. Within a fixed sample volume, however, the increased concentration will also lead to more particles that produce heat. In order to study the heating power in a fixed sample volume for different particle concentrations Fig. 6 shows the quantity $A c$, characterizing the volume specific absorption rate (VSAR), depending on the particle concentration $c$. For small concentrations an almost linear increase of the VSAR can be seen since dipole-dipole interactions are still very weak. At higher concentrations a maximum can be found with a further increase of particle density, leading again to a reduced VSAR. This result is of great importance since it suggests that an upper limit exists for the number of particles that should be injected into a fixed tumor volume, defined

\section{chrihaase@gmx.de}

${ }^{1}$ M. Jeun, S. Bae, A. Tomitaka, Y. Takemura, K. H. Park, S. H. Paek, and K. W. Chung, Appl. Phys. Lett. 95, 082501 (2009).

${ }^{2}$ J. M. Vargas, W. C. Nunes, L. M. Socolovsky, M. Knobel, and D. Zanchet, Phys. Rev. B 72, 184428 (2005).

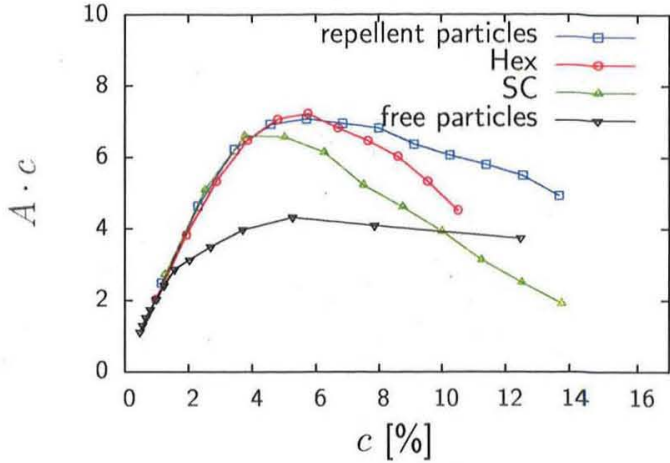

FIG. 6. (Color online) Heating power per sample volume $A c$ vs particle concentration $c$. Systems with different spatial distributions (regular simple cubic and hexagonal structures as well as random repellent and free particles) are compared. In all cases an optimal density exists.

by the dipole-dipole interactions. As can be seen from the different curves in Fig. 6, this optimal value is influenced by the different spatial distributions of the particles. For a comparison to the disordered systems (repellent and free particles) results for two ordered systems with hexagonal (Hex) and simple cubic (SC) lattice structures are also presented.

\section{CONCLUSION}

The efficiency of magnetic particle heating for hyperthermia treatment was investigated numerically. A clearly negative influence of the dipole-dipole interactions was found for increasing particle concentrations, though the study of different sample shapes showed that only in very elongated samples can a slightly increased hysteresis area be achieved through small dipole-dipole interactions. The reduced hysteresis area found for all other cases of interacting systems is in agreement with the theoretical findings of Serantes et al. ${ }^{17}$ and Burrows et $a l^{21}$ The experimental results presented by Linh et al. ${ }^{22}$ further support our findings.

In an additional investigation we showed that for the highest heating power per sample volume an optimal particle concentration exists. Such an optimal concentration maximizes the sample VSAR instead of the commonly discussed SAR. We showed that different spatial particle arrangements influence the optimal concentration values with a possible saturation behavior. Corresponding experimental results with a saturated heating power under increasing particle numbers can be found in the works of Hilger et al. ${ }^{23,24}$ In a practical treatment scenario finding the optimal particle concentration can be of great importance for an efficient application of magnetic hyperthermia.

${ }^{3}$ U. O. Häfeli, J. S. Riffle, L. Harris-Shekhawat, A. CarmichaelBaranauskas, F. Mark, J. P. Dailey, and D. Bardenstein, Mol. Pharmaceutics 6, 1417 (2009).

${ }^{4}$ I. Hilger, A. Kießling, E. Romanus, R. Hiergeist, R. Hergt, W. Andrä, M. Roskos, P. Weber W. Linss, W. Weitschies, and W. A. Kaiser, Nanotechnology 15, 1027 (2004). 
${ }^{5}$ R. Tietze, R. Jurgons, S. Lyer, E. Schreiber, F. Wiekhorst, D. Eberbeck, H. Richter, U. Steinhoff, L. Trahms, and C. Alexiou, J. Magn. Magn. Mater. 321, 1465 (2009).

${ }^{6}$ J. P. Fortin, F. Gazeau, and C. Wilhelm, Eur. Biophys. J. 37, 223 (2008).

${ }^{7}$ Y. Q. Zheng, C. Y. Tong, and B. Wang, Chin. Sci. Bull. 54, 2998 (2009).

${ }^{8}$ M. Johannsen, A. Jordan, R. Scholz, M. Koch, M. Lein, S. Deger, J. Roigas, K. Jung, and S. Loening, J. Endourol. 18, 495 (2004).

${ }^{9}$ I. S. Poperechny, Yu. L. Raikher, and V. I. Stepanov, Phys. Rev. B 82, 174423 (2010).

${ }^{10}$ Yu. L. Raikher, V. I. Stepanov, and R. Perzynski, Phys. B 343, 262 (2004).

${ }^{11}$ M. L. Plumer, J. van Lierop, B. W. Southern, and J. P. Whitehead, J. Phys. Condens. Matter 22, 296007 (2010).

${ }^{12}$ D. V. Berkov, N. L. Gorn, and P. Görnert, Phys. Status Solidi A 189, 409 (2002).

${ }^{13}$ R. Hergt, S. Dutz, and M. Röder, J. Phys. Condens. Matter 20, 385214 (2008).

${ }^{14}$ A. Jordan, R. Scholz, K. Maier-Hauff, M. Johannsen, P. Wust, J. Nadobny, H. Schirra, H. Schmidt, S. Deger, S. Loening, W. Lanksch, and R. Felix, J. Magn. Magn. Mater. 225, 118 (2001).
${ }^{15}$ R. Hergt, S. Dutz, and M. Zeisberger, Nanotechnology 21, 015706 (2010).

${ }^{16}$ R. E. Rosensweig, J. Magn. Magn. Mater. 252, 370 (2002).

${ }^{17}$ D. Serantes, D. Baldomir, C. Martinez-Boubeta, K. Simeonidis, M. Angelakeris, E. Natividad, M. Castro, A. Mediano, D.-X. Chen, A. Sanchez, L. I. Balcells, and B. Martinez, J. Appl. Phys. 108, 073918 (2010).

${ }^{18} \mathrm{~T}$. L. Gilbert, IEEE Trans. Magn. 40, 3443 (2004).

${ }^{19}$ D. C. Montgomery and G. C. Runger, Applied Statistics and Probability for Engineers (Wiley, Hoboken, NJ, 2010).

${ }^{20}$ U. Nowak, Annu. Rev. Comput. Phys. 9, 105 (2001).

${ }^{21}$ F. Burrows, C. Parker, R. F. L. Evans, Y. Hancock, O. Hovorka, and R. W. Chantrell, J. Phys. D 43, 474010 (2010).

${ }^{22}$ P. H. Linh, P. V. Thach, N. A. Tuan, N. C. Thuan, D. H. Manh, N. X. Phuc, and L. V. Hong, J. Phys. Conf. Ser. 187, 012069 (2009).

${ }^{23}$ I. Hilger, W. Andrä, R. Hergt, R. Hiergeist, H. Schubert, and W. A. Kaiser, Radiology 218, 570 (2001).

${ }^{24}$ I. Hilger, R. Hergt, and W. A. Kaiser, Invest. Radiol. 35, 170 (2000). 